\title{
MEDICAL STEEL FAULT PREDICTION USING DEEP LEARNING TECHNIQUES
}

\author{
A.Sheik Abdullah, \\ Assistant Professor, Department of \\ Information Technology, Thiagarajar \\ college of Engineering, Madurai, \\ Tamilnadu, India.
}

\author{
Karthikeyan Jothikumar, \\ Research Associate, Department of \\ Information Technology, Thiagarajar \\ college of Engineering, Madurai, \\ Tamilnadu, India.
}

\author{
K.R.A.Bhubesh, \\ Department of Information \\ Technology, Thiagarajar college of \\ Engineering, Madurai, Tamilnadu, \\ India.
}

\begin{abstract}
Fault detection and analysis is considered to be an important factor in industrial production for medical applications. As industrial era has evolved tons, new fault identification ways are required to differentiate faults with totally good distinctions. The superior best a production is wanted to possess, the higher fault identification methodology the factories should apply. This research work focus on the assessment and evaluation of fault detection using deep learning techniques. The evaluation is made accordingly using Deep CNN with the variants corresponding to simple CNN, Resnet, Alexnet and Vgg_16. Besides, classification accuracy is improved by parameter optimizing and sample size equalization strategy. Experimental results shows that evaluation using the proposed methods with Vgg_16 gives an improved training accuracy of about $90 \%$ and validation accuracy of about $87 \%$. This proves that fault detection and analysis in medical equipments and transplanting devices can be efficiently identified for better treatment and device management.
\end{abstract}

Keywords-Deep learning, Image Augmentation, Neural Networks, Fault Detection, Medical devices.

\section{INTRODUCTION}

Fault detection and analysis is becoming an important phenomenon in the forth coming days. Medical fault prediction and analysis is needed in dayto-day surgical incorporation and its applications [1]. The classification of fault and its mechanism need to be considered more important because the materials used for incorporation varies accordingly with the treatment concerned. Managing and maintaining the fault types with materials, cost and its waste level we can improve the quality of the device or the equipment that is to be used [2]. Similar measures corresponding to the recycling of materials will also happen corresponding to the medical or surgical fault diagnosis [3].
The operational environments should be made flexible with regard to the fault and its diagnosing principles. Industrial production is also threatening to the medical environment for fault methods and makes things fine distinctions [4]. The quality should not be compromised at any situations with regard to the production and analysis methods [5]. Parameter estimation and optimizing the quality of the materials used makes the medical steel plate production to have a good and gatherable environment for the industry to produce the material [6].

Traditional learning algorithms in machine learning platform have its restrictions in different domains of real-time engineering applications. When considering the medical domain algorithms focusing on deep learning models has a good impact and predictive nature with the application considered [7]. If this is made in novel practice for the prediction of fault rate in steel prediction then the exact relevance and its coordinates for the material can be determined in advance [8]. The dimension, shape, size, and quality of the material are the most important parameters for any material to test for evaluation. This if made clear and concise then the production of good quality material can be adhered at all the stages of usage for medical practice [9].

Deep learning models are mainly used in medical applications in order to improve the prediction and to make diagnosis in a better way [10]. Researchers and clinical investigators use the concept of deep learning to predict and enhance the nature of disease, risk factors, and medical diagnostic compliances [11]. At certain stages, the realm of heart failure rates and its detective models can be made accordingly with the deep learning techniques. Algorithms such as 
convolutional neural network, vector classifiers, and tree-based evaluation methods are the most used algorithmic models for prediction and classification in medical informatics [12]. This research work focus on the assessment and evaluation of efficiency of medical devices specifically on steel plate that is used for surgical practice. The entire process has been modelled using Deep learning techniques upon statistical evaluation and analysis.

\section{LITERATURE REVIEW}

The work by the authors Isermann [1] strategies are based, e.g., on boundary assessment, equality conditions or state eyewitnesses. Additionally signal model methodologies were created. The objective is to produce a few manifestations demonstrating the distinction among ostensible and defective status. In view of various side effects deficiency analysis techniques follow, deciding the flaw by applying grouping or derivation strategies. This commitment gives a short presentation into the field and gives a few applications for an actuator, a traveler vehicle and an ignition motor.

The work by the authors Dong [2] lot of channels is planned where each channel intends to mutually appraise the framework states and a particular conceivable issue. Upper limits of the assessment blunder covariance's are acquired in the concurrent presence of the linearization mistakes and decentralized occasion set off transmissions, and afterward the channel gains are determined to limit such limits. The channels are planned in a recursive manner and subsequently the calculation is relevant for online execution. At the point when a shortcoming is identified, the channel with the least remaining is viewed as the one comparing to the real flaw and its yield can be viewed as the states and issue assessment. The adequacy of the proposed strategy is represented by a reenactment model.

The work by the authors Good et al [17] introduces an algorithm that greatly reduces the overall size of the PCA problem by breaking the analysis of a large number of variables into multiple analyses of smaller uncorrelated blocks of variables. From the statistical summary it has been observed that the compatibility of PCA found to be good at the implementation level of all variables.
Similarly, the authors Yin et al [3] proposed an approach which is different from the standard PCA- and PLS-based techniques which rely on mean-extraction for residual generation; the proposed CVA-based scheme takes process dynamics into account as well. Also, the significance and the corresponding property provide an improvement when compared to that of the benchmark process.

The work by the authors Breiman et al [15] tests on genuine and mimicked informational collections utilizing arrangement and relapse trees and subset determination in direct relapse show that stowing can give significant increases in exactness. The crucial component is the shakiness of the forecast strategy. In the event that annoying the learning set can cause huge changes in the indicator developed, at that point stowing can improve precision.

The work by the authors Bewick et al [18] presents strategic relapse, which is a strategy for demonstrating the reliance of a paired reaction variable on at least one illustrative factors. Constant and clear cut illustrative factors are thought of.

The work made by the author Widodo [4] manages the utilization of the previously mentioned classifiers for flaw finding of a concoction cycle containing a ceaseless mixed tank reactor and a warmth exchanger. The outcomes show a predominant characterization execution of the help vector machine versus the chose fake neural organization. Also, the help vector machine classifier is extremely delicate to the correct determination of the preparation boundaries. It is demonstrated that the use of hereditary calculation for ideal determination of these boundaries is doable and can assist with improving the help vector machine classifier execution. Similarly, Terzi et al [20] provided a scientific categorization for arranging classifiers is introduced. Another meta-classifier, Meta-Consensus, with a establishment in both agreement hypothesis and the hypothesis of autonomous appointed authorities, is presented.

The work by the author Basheer [5] manages the utilization of the previously mentioned classifiers for flaw finding of a concoction cycle containing a ceaseless mixed tank reactor and a warmth exchanger. The outcomes show a predominant characterization execution of the help vector machine versus the chose 
fake neural organization. Also, the help vector machine classifier is extremely delicate to the correct determination of the preparation boundaries. It is demonstrated that the use of hereditary calculation for ideal determination of these boundaries is doable and can assist with improving the help vector machine classifier execution. In this research work we explicitly focus on the evaluation of the steel plate which is specifically used in surgical process. The implementation is preceded with the deep learning techniques upon statistical evaluation.

\section{PROPOSED METHODOLOGY}

\section{A. Data Collection}

Data corresponding to the Northeastern University (NEU) is collected which specifically corresponds to the surface defect focusing on six different surfaces. The defect is analyzed with regard to the hot-rolled strip of steel with rolled-in scale, crazing, pitted, scratches, patches and inclusion surfaces. The database corresponds to 1800 grayscale images and 300 samples of each of the six forms of defects on each of the surface. The observed images clearly depict the intraclass defects focusing on horizontal, vertical and slanting scratch surfaces. But, the inter-class defects have similar aspect of defect focusing on scale, crazing and regions of pitted surface. From the surface defect database there exists two challenges such as the appearance of the defect and the influence of enlightened patterns when the quality of the material changes.

\section{B. Data Preprocessing}

The dataset used for the fault detection is the preprocessed image dataset. So we applied some noise reduction alone to use it in our algorithm. The image is in bmp format and image is preprocessed as grey scale image. The reason to use bmp format and grey scale image is bit map format identifies the spot of faults in steel images more precisely than jpg format. The grey scale is more precise for fault identification than coloured images.

III.B.1 Basic Image Data Analysis: Basic image data analysis show the image basic properties like image size, RGB value calculation and heuristic natures of image to train without much discrepancy.
Type of the image: 〈class 'imageio.core.util.Array'>

Shape of the image : $(200,200)$

Image Hight 200

Image Width 200

Dimension of Image 2

Figure 1. Data Preprocessing

\section{Image Augmentation}

The data provided from the source was only in limited numbers. For efficient training and classification of faults we need more images for training and testing. So we used some automation on image augmentation which will generate images based upon given needs like image size shape and format etc.

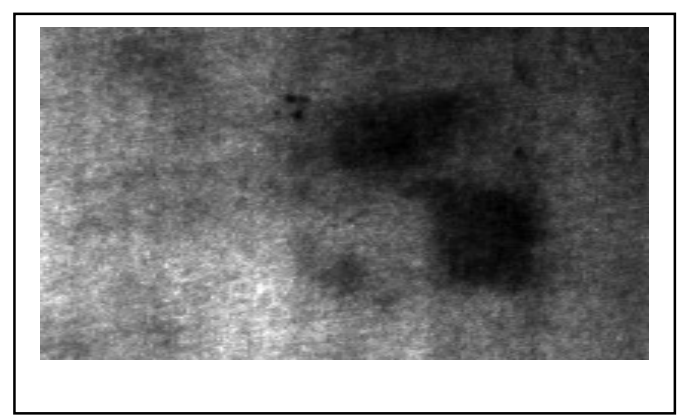

Figure 2. Original Image

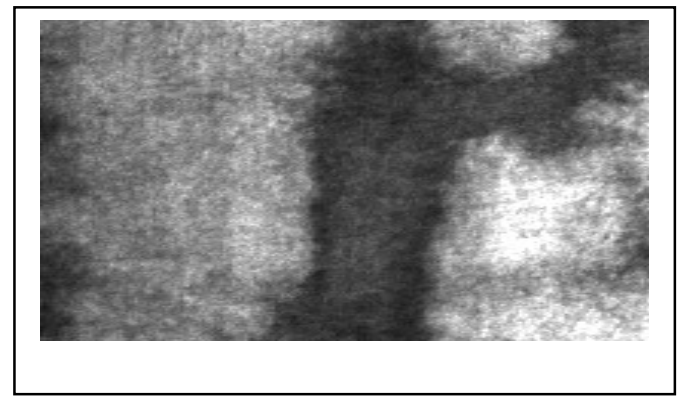

Figure 3. Augmented Image

From above 2 images it is clear that the augmented images are matched upto $95 \%$ with original image. So we augmented up to 1000 images for each class of fault in the given dataset. The original and augmented images are structured in Figure 2 and Figure 3. 


\section{EXPERIMENTAL RESULTS AND DISCUSSION}

\section{a. Simple Deep CNN}

In this the architecture is pretty old architecture that is Deep CNN. It has 6 Convolution layer and 1 output layer, each convolution layers uses a filters of 30 $\mathrm{X} 30$ which is better of viewing the fault section in grey scale training images. The kernel size used in here is 3 $\mathrm{X} 3$ and activation function in all layers is tanh, which responds well for minor pixel changes it does not show many deviations but with major steep pixel changes the activation performs well. The following Figure 4 signifies the processed image for steel fault rate.

The image data is converted to numpy arrays and the numpy arrays fed into these convolution layers for training. The pixels values of fault spots are trained inside the architectures and the model is validated against the validation data.

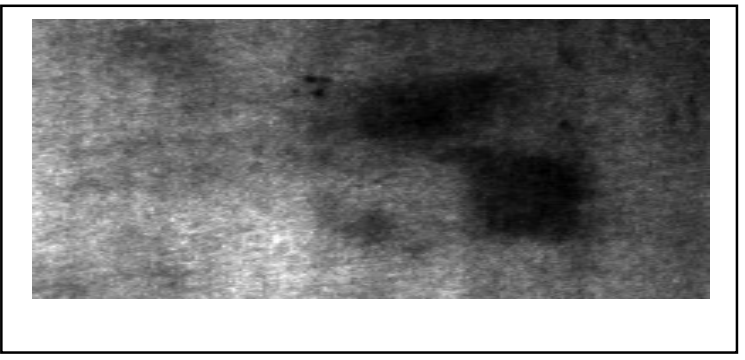

Figure 4. Processed Image (Steel fault detection)

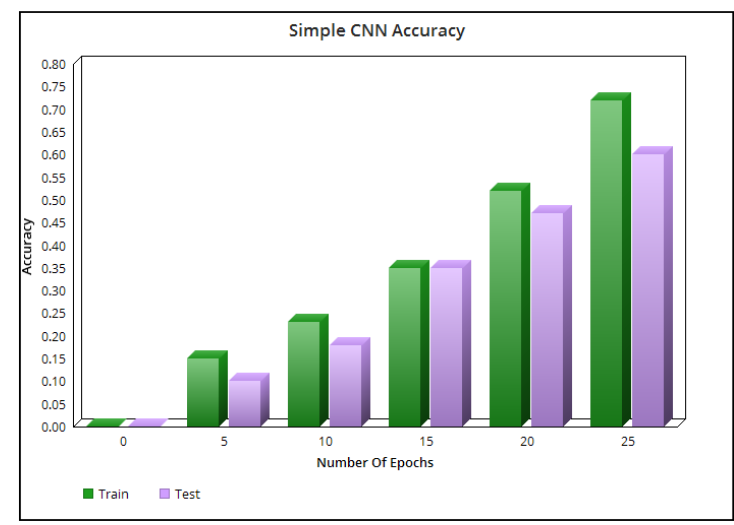

Figure 5. Accuracy Graph for Simple Deep Cnn

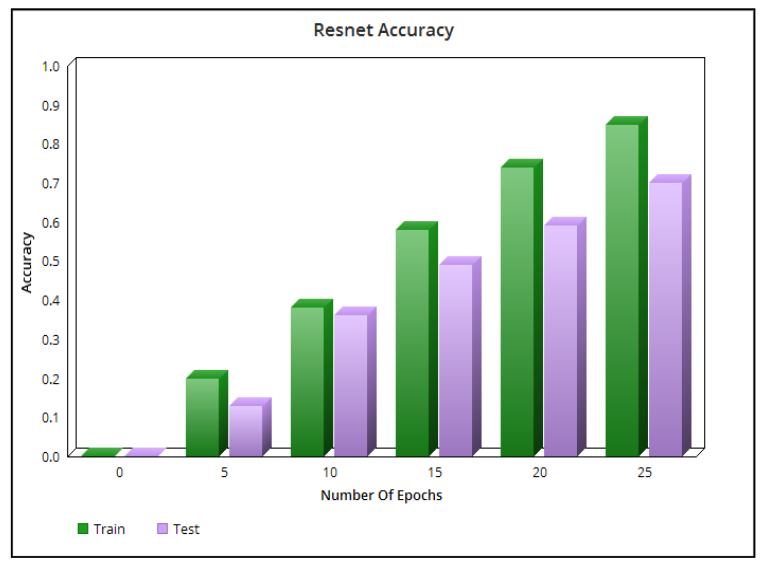

Figure 6. Accuracy Graph for Resnet

\section{b. Resnet}

In this 16 convolution layers are used for more precise insights of pixel values each layer uses 512 filters with kernel size of $3 \times 3$. Here the image is trained as it is without conversion of numpy arrays. Since resnet Architecture will take for pixels with 2 of its convolution layers. The activation function used in each layer was rectified linear unit which was most efficient activation function for the resent architecture. The output layer uses the softmax as output function. Every layer is trained with minute value of pixels so for 16 convolution layers the pixel of faults will be well trained and provide greater accuracy than the simple deep cnn. The Accuracy evaluation is given in Figure 6.

\section{c. Alexnet}

In this 12 convolution architectures is used with 256 filters with kernel size of $3 \times 3$. Dropout values are used in this architecture here also the image is trained as it is. Alexnet uses softmax activation function in its convolution layers in this recurrent unit is attached so that some pixel conditions are maintained for the future remembrance which makes this architecture as most powerful one.

The output layer uses the relu activation function which converts and gives the normalised for higher grey scale values. The accuracy is depicted in Figure 7. 


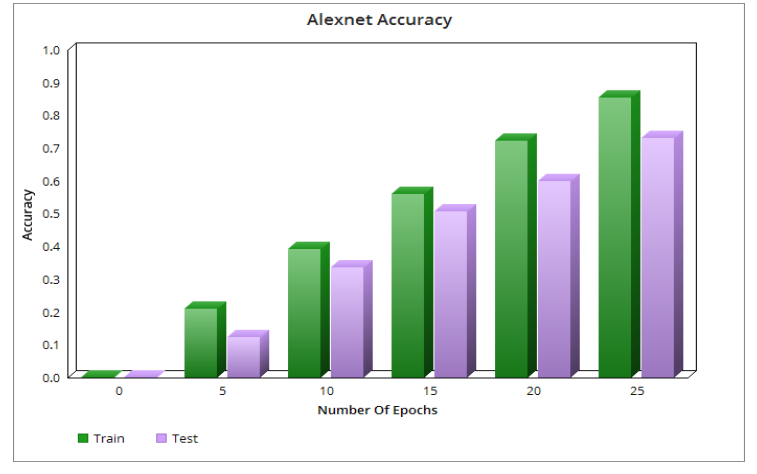

Figure 7. Accuracy Graph for Alexnet

\section{d. Vgg}

Vgg uses the 16 and 32 convolution layers for greater accuracies. Here the vgg 16 Architecture is used so 16 convolution layers are used the activation function used is softmax at each layer. The image is converted into numpy arrays and then trained and validated with the model. The accuracy is depicted in Figure 8. The output layer uses the relu activation function with some regularization to avoid unnecessary pixels. The comparison among the accuracy of the model is depicted in Table 1.

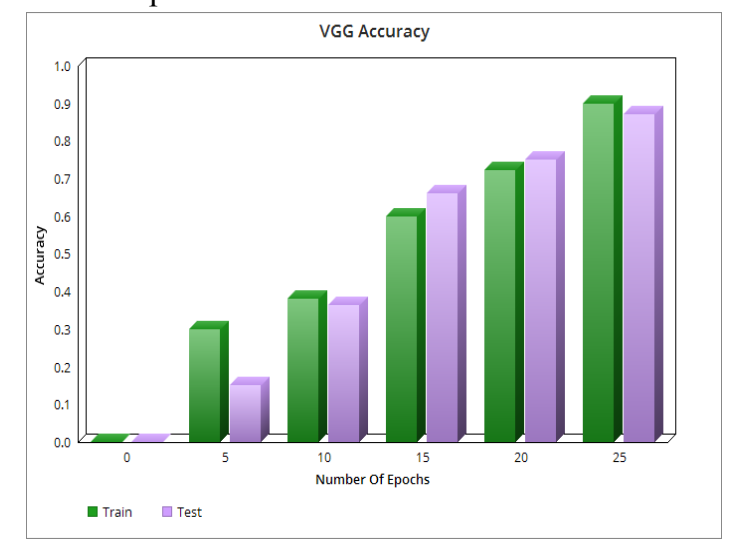

Figure 8. Accuracy Graph for Vgg

Table 1. Experimental results

\begin{tabular}{|c|c|c|}
\hline Architecture & $\begin{array}{c}\text { Training } \\
\text { Accuracy }\end{array}$ & $\begin{array}{c}\text { Validation } \\
\text { Accuracy }\end{array}$ \\
\hline Simple CNN & $72 \%$ & $60 \%$ \\
\hline RESNET & $85 \%$ & $70 \%$ \\
\hline ALEXNET & $85.4 \%$ & $73 \%$ \\
\hline VGG_16 & $90 \%$ & $87 \%$ \\
\hline
\end{tabular}

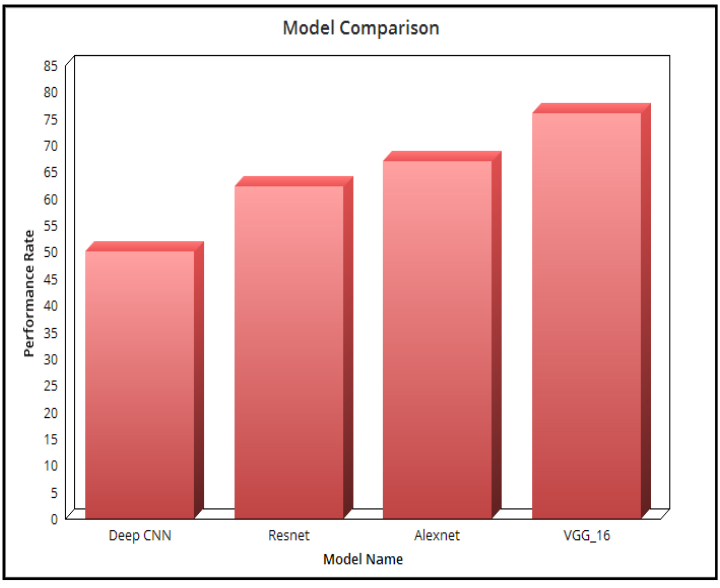

Figure 9. Model comparison and analysis

\section{CONCLUSION AND FUTURE WORK}

Fault identification and analysis in devices and equipments is one of the most frequently occurring phenomenon's in production technology. This research work focus on the assessment and evaluation of fault rate in steel plates using the variants of deep $\mathrm{CNN}$. The variants involved are simple $\mathrm{CNN}$, Resnet, Alexnet and Vgg_16. Among the four different variants of Deep CNN vgg_16 provided a good impact in fault identification and validation accuracy. This if deployed in production engineering can fix the problems and issues related to fault detection and analysis. Moreover manual intervention collection of data for classification algorithms is literally reduced since the image is directly trained with help of CNN. In future works we will enhance the operational facilities of this idea to all metal sheets with greater accuracy and less time complexity.

\section{REFERENCES}

[1] Isermann, R. Model-based fault-detection and diagnosis - status and applications. Annu. Rev. Control 29 (1) pp. 71-85, 2005.

[2] Dong, H., Wang, Z., \& Gao, H. Fault detection for markovian jump systems with sensor saturations and randomly varying nonlinearities, Circuits and Systems I: Regular Papers. IEEE Transactions on 59 (10) pp. 2354-2362, 2012.

[3] Yin, S., Ding, S.X., Haghani, A., Hao, H., \& P. Zhang. A comparison study of basic data-driven fault diagnosis and process monitoring methods on the benchmark Tennessee Eastman process. J. Process Control 22 (9) pp. 1567-1581, 2012.

[4] Widodo, A., \& Yang, B.S. Support vector machine in machine condition monitoring and fault diagnosis. Mech. Syst. Signal Process. 21 (6) pp. 2560-2574, 2007. 
[5] Basheer, I., \& Hajmeer, M. Artificial neural networks: fundamentals, computing, design, and application, J. Microbiol. Methods 43 (1) pp. 3-31, 2000

[6] Yin, S., Ding, S., Xie, X., \& Luo, H. A review on basic datadriven approaches for industrial process monitoring, IEEE Trans. Ind. Electron. 61 (11) 6418-6428, 2014.

[7] Du, W., \& Zhan, Z. Building decision treeclassifieronprivatedata, in: Proceedings of the IEEE International Conference on Privacy, Security and Data Mining, vol. 14, Australian Computer Society, Inc., pp. 1-8, 2002.

[8] Zou, H., Hastie, T., \& Tibshirani, R. Sparse principal component analysis, J. Comput. Graphical Stat. 15 (2) pp. $265-$ 286, 2006.

[9] Braga, J., Heuze, Y., Chabadel, O., Sonan, N., \& Gueramy, A. Non-adult dental age assessment: correspondence analysis and linear regression versus Bayesian predictions, Int. J. Legal Med. 119 (5) pp. 260-274, 2005.

[10] Russell, E.L., Chiang, L.H., \& Braatz, R.D. Fault detection in industrial processes using canonical variate analysis and dynamic principal component analysis, Chemom. Intell. Lab. Syst. 51 (1) pp. 81-93, 2000.

[11] Bach, F.R., \& Jordan, M.I. Kernel independent component analysis, J. Mach. Learn. Res. 3 pp. 1-48, 2003.

[12] Yin, S., Zhu, X., \& Kaynak, O. Improved pls focused on key performance indictor related fault diagnosis, IEEE Trans. Ind. Electron. 2014.

[13] Amit ,Y., \& Geman, D. "Shape quantization and recognition with randomized trees," Neural Comput., vol. 9, no. 7, pp. 1545-1588, 1997.

[14] Breiman, L. "Random forests," Mach. Learn., vol. 45, no. 1, pp. 5-32, 2001.

[15] Breiman, L. "Bagging predictors," Mach. Learn., vol. 24, no. 2, pp. 123-140, 1996.

[16] Dong, Y., Du, B., \& Zhang ,L."Target Detection Based on Random Forest Metric Learning," in IEEE Journal of Selected Topics in Applied Earth Observations and Remote Sensing, vol. 8, no. 4, pp. 1830-1838, April 2015.

[17] Good, R.P., Kost, D., \& Cherry, G.A. "Introducing a Unified PCA Algorithm for Model Size Reduction," in IEEE Transactions on Semiconductor Manufacturing, vol. 23, no. 2, pp. 201-209, May 2010.

[18] Bewick, V., L. Cheek andJ. Ball, Statistics review 14: Logistic regression. Crit Care 9: 112-118. DOI: 10.1186/cc3045, 2005.

[19] Breiman, L., Classification and Regression Trees. 1st Edn., Wadsworth International Group, Belmont, ISBN-10: 0534980538 pp: $358,1984$.

[20] Buscema, M., S. Terzi and W. Tastle, A new metaclassifier. Proceedings of the North American Fuzzy Inform Processing Society, Jul. 12-14, IEEE Xplore Press, Toronto, pp: 1-7. DOI: 10.1109/NAFIPS.2010.5548298, 2010.

[21] Chaudhuri, B.B. and U. Bhattacharya, Efficient training and improved performance of multilayer perceptron in pattern classification. Neurocomputing, 34: 11-27. DOI: 10.1016/S0925-2312(00)00305-2, 2000.

[22] Dong, L., D. Xiao, Y. Liang and Y. Liu, Rough set and fuzzy wavelet neural network integrated with least square weighted fusion algorithm based fault diagnosis research for power transformers. Elec. Power Syst. Res., 78: 129-136. DOI: 10.1016/J.EPSR.2006.12.013, 2008.

[23] Eslamloueyan, R., Designing a hierarchical neural network based on fuzzy clustering for fault diagnosis of the Tennessee-
Eastman process. Applied Soft Comput., 11: 1407-1415. DOI: 10.1016/J.ASOC.2010.04.012, 2011.

[24] Haykin, S.S., Neural Networks: A Comprehensive Foundation. 1st Edn., Macmillan, New York, ISBN-10: 0023527617, pp: 69, 1994.

[25] Zhang, Y., Jiang, J, Bibliographical review on reconfigurable fault-tolerant control systems. Annual Rev. Control, 32: 229252. DOI: 10.1016/J.ARCONTROL.2008.03.008, 2008.

[26] Maurya, M.R., Rengaswamya, R., Venkatasubramanian, V., Fault diagnosis using dynamic trend analysis: A review and recent developments. Eng. Appli. Artif. Intell., 20: 133-146. DOI: 10.1016/j.engappai.2006.06.020, 2007.

[27] Lo, C.H., Wong, Y.K., Rad, A.B., Chow, K.M., Fusion of qualitative bond graph and genetic algorithms: A fault diagnosis application. ISA Trans., 41: 445-456. 10.1016/S00190578(07)60101-3, 2002. 